\title{
ALCOHOL Y JUVENTUD ¿EXISTEN DIFERENCIAS EN CONSUMO DE HOMBRES Y MUJERES SEGÚN EDAD DE INICIO?
}

\author{
Cristina Giménez-García \\ gimenezc@uji.es \\ Estefanía Ruiz-Palomino \\ Beatriz Gil-Juliá \\ Juan Enrique García-Nebot \\ Rafael Ballester-Arnal \\ Salusex-Unisexsida. Departamento de Psicología Básica, Clínica y Psicobiología. \\ Facultad de Ciencias de la Salud. Universitat Jaume I de Castellón.
}

Fecha de Recepción: 3 Febrero 2018

Fecha de Admisión: 10 Abril 2018

\section{RESUMEN}

En la adolescencia, el consumo de alcohol supone uno de los problemas de salud más acuciantes. Sin embargo, tanto los adolescentes como la sociedad, muestran una baja percepción de gravedad. En este contexto, la edad de inicio podría establecer diferencias en los patrones de consumo, facilitando su consolidación. Por ello, se analiza si existen diferencias entre aquellas personas que iniciaron su consumo antes y después de los 14 años, en cuanto a la frecuencia de consumo, los beneficios percibidos y los problemas ocasionados. Con tal fin, 894 jóvenes españoles entre 18 y 22 años (33,6\% hombres y 67,3\% mujeres), cumplimentaron un cuestionario sobre hábitos de salud dirigido a población joven. Según los resultados, el 99,4\% consume alcohol en el momento actual y más de un tercio comenzó antes de los 14 años. Los hombres iniciarían antes tanto el consumo, como las primeras borracheras (entre los 10 y 12 años), mostrando diferencias estadísticamente significativas con las mujeres, en el primer caso $\left(\mathrm{Chi}^{2}=15,66 ; p \leq, 008\right)$. En líneas generales, tanto en hombres como en mujeres, se observa cómo las personas que iniciaron el consumo antes de los 14 años, muestran una mayor frecuencia en el mismo. En algunos casos, como en la ingesta diaria de cerveza, llegan a doblar el consumo de los que lo iniciaron después. Además, reportan en mayor medida haber sufrido consecuencias negativas por el alcohol, así como percibir beneficios relacionados con su consumo como sentirse más seguros, conocer más fácilmente a personas, sentir euforia o combatir el aburrimiento con más facilidad. Así pues, los resultados apoyan una mayor exposición al riesgo, conductual y actitudinal, entre aquellas personas que comenzaron antes el consumo. De esta forma, se vuelve a poner de manifiesto la importancia de retrasar el consumo del alcohol y, en consecuencia, de gestionar planes preventivos dirigidos a edades más tempranas. 
Palabras clave: consumo alcohol; jóvenes; inicio temprano; beneficios percibidos; frecuencia de consumo

\section{ABSTRACT \\ Alcohol and youth. are there differences in the consumption of men and women according to their beginning of consumption?}

In adolescence, alcohol consumption is one of the most demanding health problems. However, both adolescents and society show a low perception of seriousness. In this context, the beginning of consumption might establish differences in patterns, facilitating their consolidation. Therefore, it is analyzed if there are differences between those people who started their consumption before and after 14 years, in terms of frequency of consumption, perceived benefits and problems. For this purpose, 894 young Spaniards between 18 and 22 years old (33.6\% men and $67.3 \%$ women), completed a questionnaire about health habits aimed at young people. According to results, $99.4 \%$ consume alcohol at the present and more than a third percent started before age 14. Men would begin both earlier consumption and their first drunkenness (between 10 and 12 years), showing statistically significant differences compared to women, for the first one $\left(\mathrm{Chi}^{2}=15.66 ; \mathrm{p} \leq, 008\right)$. In general, in both men and women, it is observed how people who started the consumption before the age of 14 show a greater frequency. In some cases, as in the daily intake of beer, they double the consumption of those who started it later. In addition, they report more damages due to alcohol consumption, as well as perceiving benefits related to their consumption such as feeling more secure, meeting people more easily, feeling euphoria or fighting boredom more easily. Thus, the results support a greater risk exposure, behavioral and attitudinal, among those people who started drinking earlier. In this way, it is supported the importance of delaying the consumption of alcohol and, consequently, managing preventive plans aimed at younger ages.

Keywords: alcohol consumption; young people; early-onset drinking; perceived benefits; frequency of consumption

\section{ANTECEDENTES}

El consumo de alcohol supone uno de los hábitos perjudiciales para la salud más extendidos entre la población joven y adolescente (Observatorio Español de Drogas y Adicciones, 2017; Plan Nacional Sobre Drogas, 2018). De hecho, alrededor del 75\% de personas, entre 14 y 18 años, informa haberlo consumido alguna vez, siendo los 13 años la edad promedio de inicio. Más de un $50 \%$ informa consumirlo de manera más habitual los fines de semana, aunque alrededor del $18 \%$ también lo hace entre semana (Plan Nacional Sobre Drogas, 2018). Además, un tercio de jóvenes entre 15 y 24 años, reconoce haber tenido alguna borrachera en el último año, con complicaciones derivadas del consumo del alcohol llegando, en algunos casos, a la intoxicación etílica (Observatorio Español de Drogas y Adicciones, 2017). Más allá de estas cifras, algunos autores alertan del riesgo de los patrones de consumo que, en alrededor de un 10\% de los adolescentes (entre 15 y 18 años), podrían presentar características cercanas a los trastornos por abuso del alcohol, vinculadas al consumo imprudente, la tolerancia o la interferencia clínicamente significativa (Ballester, Gil \& Guirado, 2000; Secades-Villa, López-Núñez, Fernández-Artamendi, Weidberg \& Fernández-Hermida, 2013).

Al mismo tiempo, la literatura científica alerta de los perjuicios biopsicosociales producidos por el alcohol. En concreto, algunos estudios destacan en qué medida el consumo abusivo de alcohol podría asociarse a ciertos daños neurocognitivos que dificultarían, entre otros, la toma de decisión de tareas cotidianas y los procesos de aprendizaje (West, Maynard \& Leasure, 2018). Asimismo, el uso de alcohol también ha sido relacionado con otros hábitos de riesgo como el consumo de otras sustancias o la conducta sexual insegura, lo que multiplicaría la exposición al riesgo de la población 
joven y adolescente (Jacobus \& Tapert, 2013; White \& Hingson, 2014). Igualmente, el consumo de alcohol se ha identificado como un facilitador de problemas psicológicos (Abrams et al., 2018), así como de las conductas violentas (Lightowlers, 2017).

Esta situación empeora si se tiene en cuenta la edad temprana de inicio y las consecuencias que podrían derivarse del mismo (Gil \& Ballester, 2002). De esta forma, más allá de los problemas que a corto plazo podría ocasionar, a medio y largo plazo, el inicio temprano parece incrementar el riesgo de padecer ciertos problemas psicológicos (Carbia, Corral, García, Cadaveira \& Caamaño, 2016) y dificultades de adaptación social, así como provocar la merma de la calidad de vida (Donoghue et al., 2017). Algunos autores, también lo han relacionado con una mayor probabilidad de padecer trastornos derivados del consumo de alcohol (Dawson, Goldstein, Chou, Ruan, \& Grant, 2008). En este sentido, un inicio temprano podría facilitar que, el consumo de alcohol intensivo realizado en la pre-adolescencia, fuera seguido por un patrón de consumo de riesgo en la juventud (Colder, Shyhalla \& Frndak, 2018; Mota et al., 2010).

En línea con propuestas contrastadas como la Teoría de la Acción Razonada (Lin, 2016), las motivaciones del uso del alcohol parecen ser uno de los factores importantes en el patrón de consumo que realizan adolescentes y jóvenes (Ballester \& Gil, 2009). En concreto, según Labhart, Kuntsche, Wicki y Gmel (2017), en la medida en que la ingesta de alcohol está relacionada con motivaciones de orden social (por ejemplo, una mayor facilidad para conocer gente), así como las relacionadas con la mejora personal (por ejemplo, sentirse bien con uno mismo) 0 el afrontamiento de situaciones (por ejemplo, olvidarse de los problemas), habría una mayor probabilidad de que el patrón de consumo presentara características de riesgo, así como de experimentar consecuencias negativas derivadas del mismo. Sin embargo, más allá de la importancia de estas variables para la prevención del consumo y, en consecuencia, de los posibles daños generados por el mismo, buena parte de los estudios se centra únicamente en el análisis de las prevalencias, sin explorar aquellos factores de riesgo como las motivaciones, que podrían propiciar los patrones de consumo de mayor riesgo (Secades-Villa et al., 2013).

\section{OBJETIVOS}

Por todo lo anterior, el presente trabajo busca analizar si existen diferencias entre aquellas personas jóvenes que iniciaron su consumo antes y después de los 14 años, teniendo en cuenta tanto la frecuencia de consumo, como las motivaciones que llevan a usar el alcohol y los problemas que les ha ocasionado su consumo. De igual forma, se quiere valorar si existen diferencias al respecto tanto en hombres como en mujeres.

\section{PARTICIPANTES}

En el estudio participaron 894 jóvenes entre 18 y 22 años, procedentes de la Universitat Jaume I de Castellón. Según el centro de estudios, el 37,4\% procedían de la Facultad de Ciencias Humanas y Sociales, el $21,4 \%$ de la Facultad de Ciencias Jurídicas y Económicas, el 19,1\% de la Facultad de Ciencias de la Salud, el 20,8\% de la Escuela de Tecnología y Ciencias Experimentales y el 1,2\% de la Escuela de Doctorado. En función del sexo, el 33,6\% eran hombres y el 67,3\% mujeres.

\section{MÉTODO.}

\section{Instrumento}

El Cuestionario de Estilo de Vida y Salud para Población Joven (Giménez-García \& BallesterArnal, 2017) evalúa, a través de sus 97 preguntas, factores de riesgo para la salud en ámbitos como la alimentación y el ejercicio físico, el consumo de sustancias, la imagen corporal, la sexualidad, el 
uso de internet y móvil, los hábitos de sueño y la seguridad vial. Este instrumento examina, a través de formato de respuesta dicotómico, escala Likert y elección múltiple, variables como la percepción de riesgo, la intención de conducta, las motivaciones para realizar conductas saludables y la realización de las propias conductas.

En concreto, se tuvieron en cuenta ocho ítems relacionados con el consumo del alcohol, que valoran tanto la conducta de consumo, como la edad de inicio, la frecuencia de consumo de determinadas bebidas alcohólicas, las motivaciones para hacerlo, la percepción de riesgo y si se han experimentado daños ocasionados por el consumo.

\section{Procedimiento}

El estudio forma parte de una investigación más amplia dirigida a analizar los estilos de vida de la población joven universitaria.

Para ello, una vez diseñado el instrumento, se administró vía telemática a través de canales online internos de la propia universidad, garantizando el anonimato y la confidencialidad. Una vez tramitada la autorización de la Comisión Deontológica de la Universidad, todos los participantes recibieron un correo electrónico en el que se informaba de la investigación, así como de su carácter voluntario. En el propio mail, también se incluía el enlace a la encuesta. Una vez los participantes accedían a dicho enlace, se encontraban con una breve reseña sobre el objetivo del estudio, así como con la necesidad de dar su consentimiento informado. Seguidamente, los participantes podían acceder a la encuesta que era cumplimentada en un tiempo aproximado de 30 minutos. Una vez obtenidos los resultados, se realizaron análisis descriptivos y diferenciales, para conocer tanto las prevalencias generales como las posibles diferencias en función de la edad de inicio de consumo del alcohol, en hombres y mujeres.

\section{RESULTADOS}

\section{Descriptivos generales y análisis diferenciales entre hombres y mujeres en el consumo de alco- hol}

El 99,4\% de los jóvenes ha consumido alcohol, sin mostrar diferencias significativamente estadísticas entre el $99 \%$ de hombres y el $99,7 \%$ de mujeres que lo ha hecho $\left(\mathrm{Chi}^{2}=1,70 ; p \leq, 191\right)$. En cuanto a la edad de inicio, entre las personas que reportan haber consumido alcohol, el 32,6\% comenzó a los 14 años 0 antes, siendo el 67,4\% el que comenzó después de dicha edad. El inicio temprano, resulta más frecuente entre los hombres que entre las mujeres, tal y como muestra la figura 1, alcanzando diferencias estadísticamente significativas (Chi $\left.{ }^{2}=15,66 ; p \leq, 008\right)$.

Figura 1. Distribucion de la edad de inicio de consumo de alcohol en hombres y mujeres

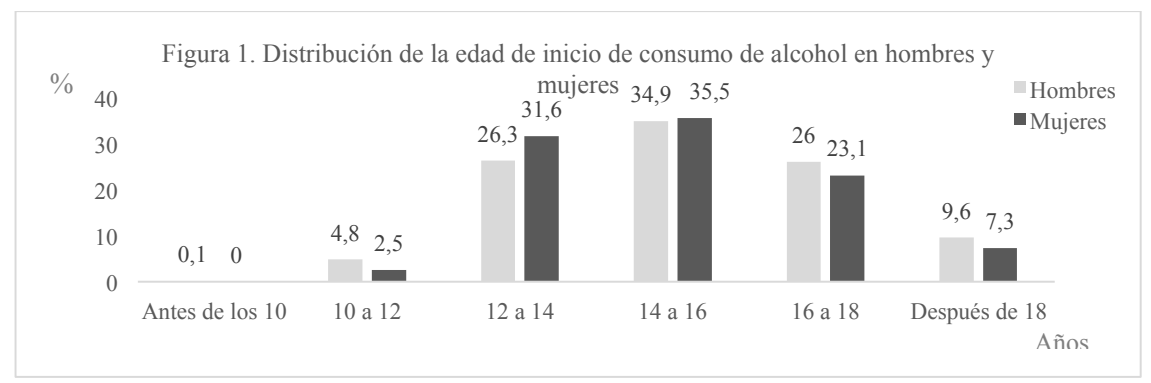


Respecto a los episodios de borracheras, el $89,7 \%$ de hombres y el $88,5 \%$ de mujeres, informa haberlas experimentado. Dicha situación, tal y como muestra la figura 2, también resulta más frecuente entre los hombres existiendo, de nuevo, diferencias estadísticamente significativas $\left(\mathrm{Chi}^{2}=14,94 ; \mathrm{p} \leq, 002\right)$.

Figura 2. Distribucion de la frecuencia de borracheras en hombres y mujeres

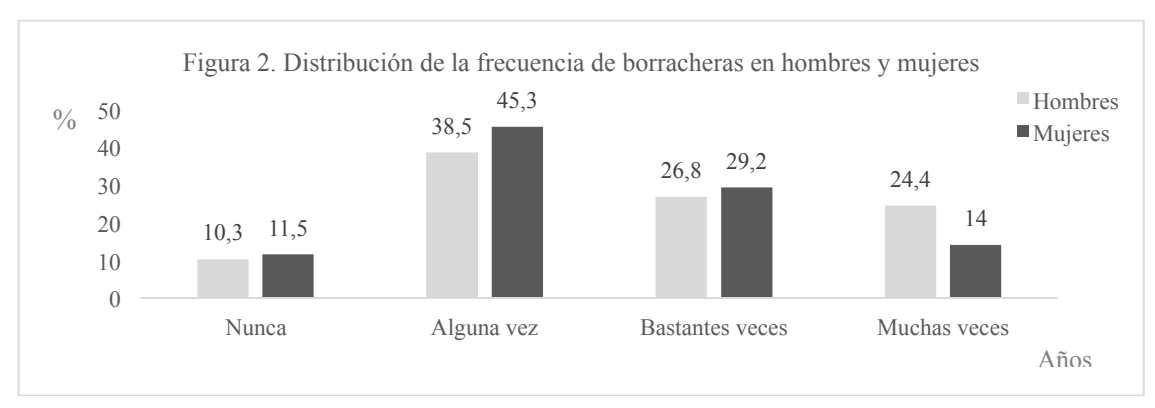

En cuanto a la edad de la primera borrachera (ver figura 3), también se observa un inicio más temprano entre los hombres, si bien no existen diferencias significativas en este caso $\left(\mathrm{Chi}^{2}=8,74\right.$; $p \leq, 068)$.

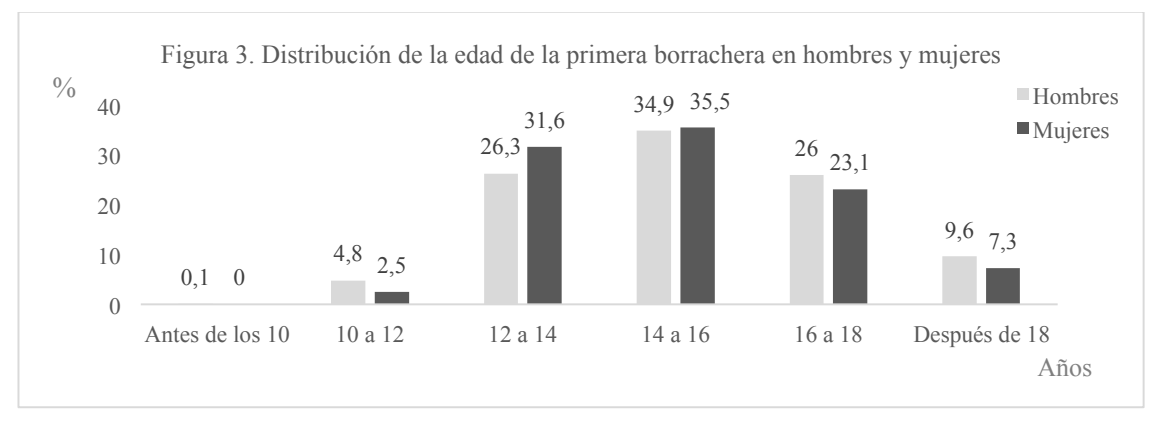

En relación al consumo de los últimos 12 meses, según el tipo de bebida, se observa cómo la mayoría de personas ha consumido bebidas destiladas, así como cerveza. De igual forma, más de la mitad de la muestra ha consumido vino, cava o bebidas similares y carajillos. En la mayoría de los casos, tal y como muestra la tabla 1, las diferencias muestran un mayor consumo entre los hombres, siendo significativas a nivel estadístico en todos los casos menos en el consumo de vino, cava 0 similares. 


\section{ALCOHOL Y JUVENTUD ¿EXISTEN DIFERENCIAS EN CONSUMO DE HOMBRES Y MUJERES SEGÚN EDAD DE INICIO?}

Tabla 1. Porcentaje de hombres y mujeres que han consumido alcohol en los últimos 12 meses, según el tipo de bebida.

\begin{tabular}{cccc}
\hline Tipo & Hombres & Mujeres & $\mathrm{Chi}^{2}(\mathrm{p})$ \\
\hline Cerveza & 86,8 & 80,4 & $15,15(, 002)$ \\
Vino, cava o similares & 61,9 & 61,5 & $7,16(, 067)$ \\
Carajillo & 62,3 & 52,7 & $20,18(, 000)$ \\
Bebidas destiladas & 88,5 & 86 & $10,72(, 013)$ \\
\hline
\end{tabular}

Respecto a las motivaciones que promueven su uso, únicamente, se encuentran diferencias entre hombres y mujeres en relación a la facilidad para combatir el aburrimiento (Chi ${ }^{2}=20,09$; $\mathrm{p} \leq, 001$ ), siendo los hombres los que más la informan (ver figura 4).

De esta forma, aunque más hombres que mujeres identifican motivaciones como la posibilidad de tranquilizarse $\left(\mathrm{Chi}^{2}=2,99 ; \mathrm{p} \leq, 084\right)$ o la facilidad para hablar $\left(\mathrm{Chi}^{2}=2,70 ; \mathrm{p} \leq, 097\right)$ y más mujeres que hombres identifican otras como sentirse seguras $\left(\mathrm{Chi}^{2}=0,40 ; p \leq, 524\right) 0$ integrarse más fácilmente en un proyecto $\left(\mathrm{Chi}^{2}=1,32 ; \mathrm{p} \leq, 250\right)$, las diferencias no son estadísticamente significativas.

Figura 4. Motivaciones para consumir alcohol de hombres y mujeres

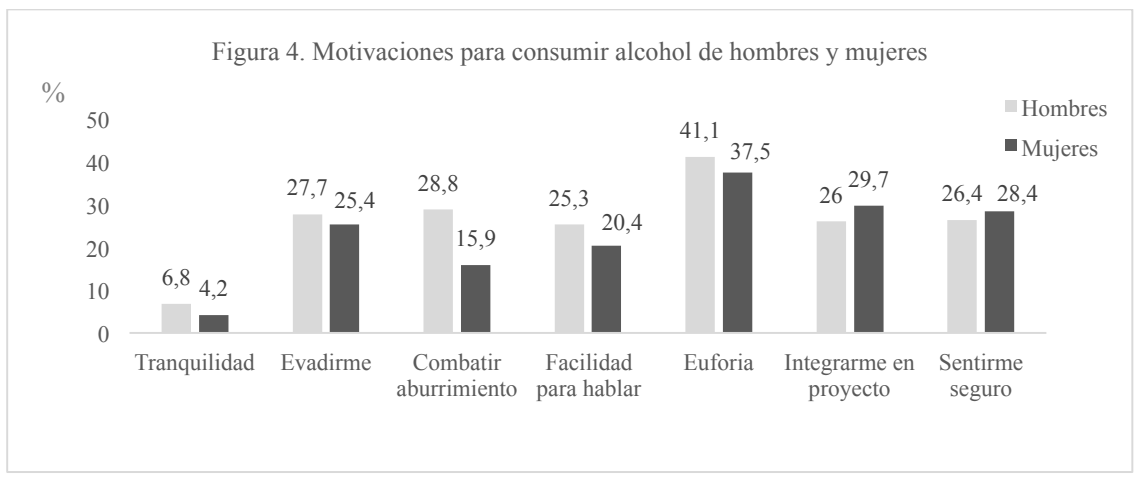

Por otro lado, se observan diferencias tanto en las consecuencias negativas experimentadas a causa del consumo del alcohol, informadas por un $25,6 \%$ de hombres y un $16,6 \%$ de mujeres $\left(C h i^{2}=9,97 ; p \leq, 006\right)$, como en la identificación del consumo del alcohol como un problema, reconocido por el $54 \%$ de los hombres y el $45,1 \%$ de las mujeres $\left(\mathrm{Chi}^{2}=7,84 ; \mathrm{p} \leq, 049\right)$.

Análisis diferenciales del consumo de alcohol, en hombres y mujeres, según la edad de inicio

En cuanto a la experiencia de las borracheras, se observan diferencias estadísticamente significativas, en hombres y mujeres, que muestran en qué medida las personas que empezaron el consumo antes de los 14 años presentan una mayor prevalencia (ver tabla 2). 
Tabla 2. Análisis diferenciales sobre la frecuencia de borracheras, en hombres y mujeres, según la edad de inicio de consumo

\begin{tabular}{cccccc}
\hline \multirow{2}{*}{ Frecuencia } & \multicolumn{2}{c}{ Hombre } & & \multicolumn{2}{c}{ Mujer } \\
\cline { 2 - 3 } \cline { 5 - 6 } \cline { 5 - 6 } & Inicio & Inicio tras & & Inicio antes & \multirow{2}{*}{ Inicio tras 14 } \\
& antes 14 & 14 & & 14,9 & 14,4 \\
Nunca & 2,3 & 13,7 & & 36 & 50,1 \\
Alguna vez & 32,6 & 41 & & 35,5 & 25,9 \\
Bastantes veces & 26,7 & 26,8 & & 22,7 & 9,6 \\
Muchas veces & 38,4 & 18,5 & & \multicolumn{2}{c}{$34,89(, 000)$} \\
$\mathrm{Chi}^{2}(\mathrm{p})$ & $18,43(, 000)$ & & \multicolumn{2}{c}{} \\
\hline
\end{tabular}

También se observan diferencias significativas a nivel estadístico, en la edad de inicio de las borracheras, tanto en hombres como en mujeres (ver tabla 3). Si bien en el caso de los hombres, se observa un inicio precoz más frecuente que en el caso de las mujeres, en ambos grupos, más de la mitad de hombres y mujeres que inician el consumo de alcohol antes de los 14 años, han experimentado una borrachera a esa edad. Por el contrario, entre las personas que inician el consumo tras los 14 años, la edad más frecuente para experimentar la primera borrachera se ubica entre los 16 y los 18 años.

Tabla 3. Análisis diferenciales en la edad de la primera borrachera, en hombres y mujeres, según la edad de inicio de consumo

\begin{tabular}{cccccc}
\hline & \multicolumn{2}{c}{ Hombre } & & \multicolumn{2}{c}{ Mujer } \\
\cline { 2 - 3 } \cline { 5 - 6 } Edad & Inicio & Inicio tras & & Inicio antes & Inicio tras \\
& antes 14 & 14 & & 14 & 14 \\
\hline $10-12$ & 11,9 & 0 & & 3,6 & 0 \\
$12-14$ & 45,2 & 0,5 & & 49,2 & 1,2 \\
$14-16$ & 23,8 & 29,9 & & 31,3 & 29,8 \\
$16-18$ & 17,9 & 46,7 & & 12,3 & 43,6 \\
$>18$ & 1,2 & 22,8 & & 3,6 & 25,4 \\
$\mathrm{Chi}^{2}(\mathrm{p})$ & $131,42(, 000)$ & & $240,19(, 000)$ \\
\hline
\end{tabular}

Respecto al tipo de bebidas consumidas, tanto en hombres como en mujeres, se observa cómo las personas que iniciaron el consumo antes de los 14 años, muestran una mayor frecuencia en el mismo. De hecho, en los hombres se alcanzan diferencias significativas para todos los tipos de bebida y, entre las mujeres, para todas a excepción del vino, cava y similares (ver tabla 4). En el caso de los chicos se observa cómo las personas que iniciaron antes de los 14, llegan a doblar el consumo de cerveza diaria y bebidas destiladas semanales que los que lo iniciaron después. De igual forma, en el caso de las mujeres, las que iniciaron el consumo antes casi doblan el consumo de bebidas destiladas semanales respecto a las que lo empezaron a consumir después y suele ser más frecuente el consumo de cerveza semanal frente a la frecuencia ocasional de las que iniciaron más tarde. 


\section{ALCOHOL Y JUVENTUD ¿EXISTEN DIFERENCIAS EN CONSUMO DE HOMBRES Y MUJERES SEGÚN EDAD DE INICIO?}

Tabla 4. Análisis diferenciales sobre la frecuencia de consumo de bebidas, en hombres y mujeres, según la edad de inicio de consumo

\begin{tabular}{|c|c|c|c|c|c|c|c|}
\hline \multirow[b]{2}{*}{$\begin{array}{l}\text { Tipo } \\
\text { bebida }\end{array}$} & \multirow[b]{2}{*}{ Frecuencia } & \multicolumn{3}{|c|}{ Hombre } & \multicolumn{3}{|c|}{ Mujer } \\
\hline & & $\begin{array}{c}\text { Inicio } \\
\text { antes } \\
14\end{array}$ & $\begin{array}{l}\text { Inicio } \\
\text { tras } 14\end{array}$ & $\begin{array}{l}\mathrm{Chi}^{2} \\
(\mathrm{p})\end{array}$ & $\begin{array}{c}\text { Inicio } \\
\text { antes } \\
14\end{array}$ & $\begin{array}{l}\text { Inicio } \\
\text { tras } 14\end{array}$ & $\begin{array}{l}\mathrm{Chi}^{2} \\
(\mathrm{p})\end{array}$ \\
\hline \multirow{4}{*}{ Cerveza } & Diario & 8,2 & 3,9 & \multirow{4}{*}{$\begin{array}{c}7,88 \\
(, 049)\end{array}$} & 4,9 & 1 & \multirow{4}{*}{$\begin{array}{l}15,93 \\
(, 001)\end{array}$} \\
\hline & Semanal & 51,8 & 43,8 & & 41 & 34,9 & \\
\hline & Ocasional & 34,1 & 36 & & 32,7 & 45,5 & \\
\hline & Nunca & 5,9 & 16,3 & & 21,5 & 18,6 & \\
\hline \multirow{4}{*}{$\begin{array}{c}\text { Vino, } \\
\text { cava, } \\
\text { similares.. }\end{array}$} & Diario & 3,6 & 0,5 & \multirow{4}{*}{$\begin{array}{l}10,36 \\
(, 016)\end{array}$} & 0,5 & 0 & \multirow{4}{*}{$\begin{array}{c}5,54 \\
(, 136)\end{array}$} \\
\hline & Semanal & 10,7 & 7,9 & & 14,6 & 10,5 & \\
\hline & Ocasional & 59,5 & 48,5 & & 50,7 & 48,7 & \\
\hline & Nunca & 26,2 & 43,1 & & 34,1 & 40,8 & \\
\hline \multirow{4}{*}{$\begin{array}{l}\text { Carajillo, } \\
\text { chupito... }\end{array}$} & Diario & 3,6 & 0 & \multirow{4}{*}{$\begin{array}{l}10,52 \\
(, 015)\end{array}$} & 0 & 0 & \multirow{4}{*}{$\begin{array}{l}10,77 \\
(, 005)\end{array}$} \\
\hline & Semanal & 14,3 & 8 & & 7,4 & 2,8 & \\
\hline & Ocasional & 50 & 52 & & 52,5 & 46,2 & \\
\hline & Nunca & 32,1 & 40 & & 40,2 & 51 & \\
\hline \multirow{4}{*}{ Destiladas } & Diario & 1,2 & 0 & \multirow{4}{*}{$\begin{array}{l}18,02 \\
(, 000)\end{array}$} & 0 & 0 & \multirow{4}{*}{$\begin{array}{l}11,68 \\
(, 003)\end{array}$} \\
\hline & Semanal & 27,4 & 10,8 & & 12,7 & 7,4 & \\
\hline & Ocasional & 66,2 & 74,9 & & 79 & 75,6 & \\
\hline & Nunca & 4,8 & 14,3 & & 8,3 & 17 & \\
\hline
\end{tabular}

En cuanto a los motivos, tanto los hombres como las mujeres que comenzaron el consumo de alcohol antes de los 14 años, muestran con mayor frecuencia motivaciones percibidas para el uso del alcohol (ver tabla 5). En el caso de los hombres, las diferencias son significativas a nivel estadístico respecto a evadirse de los problemas, tener una mayor facilidad para conversar y la percepción de combatir así el aburrimiento. En las mujeres, además de en estas dos últimas, también se observan en la facilidad para conocer gente, sentir euforia, integrarse en un proyecto y sentir seguridad en sí mismas. 
Tabla 5. Análisis diferenciales sobre los motivos de consumo, en hombres y mujeres, según la edad de inicio de consumo

\begin{tabular}{|c|c|c|c|c|c|c|}
\hline \multirow[b]{2}{*}{ Motivos } & \multicolumn{3}{|c|}{ Hombre } & \multicolumn{3}{|c|}{ Mujer } \\
\hline & $\begin{array}{c}\text { Inicio } \\
\text { antes } 14\end{array}$ & $\begin{array}{l}\text { Inicio } \\
\text { tras } 14\end{array}$ & $\begin{array}{l}\mathrm{Chi}^{2} \\
(\mathrm{p})\end{array}$ & $\begin{array}{c}\text { Inicio } \\
\text { antes } 14\end{array}$ & $\begin{array}{l}\text { Inicio } \\
\text { tras } 14\end{array}$ & $\mathrm{Chi}^{2}(\mathrm{p})$ \\
\hline $\begin{array}{l}\text { Sentirme más } \\
\text { tranquilo }\end{array}$ & 5,8 & 7,3 & $\begin{array}{c}0,20 \\
(, 651)\end{array}$ & 4,9 & 3,8 & $0,41(, 522)$ \\
\hline $\begin{array}{l}\text { Evadirme de } \\
\text { problemas }\end{array}$ & 39,5 & 22,8 & $\begin{array}{c}8,46 \\
(, 004)\end{array}$ & 25,4 & 25,4 & ,000 (,984) \\
\hline $\begin{array}{c}\text { Evitar } \\
\text { aburrimiento }\end{array}$ & 38,4 & 24,8 & $\begin{array}{c}5,48 \\
(, 004)\end{array}$ & 22 & 12,8 & $8,36(, 004)$ \\
\hline Conocer gente & 43 & 39,8 & $\begin{array}{c}0,26 \\
(, 610)\end{array}$ & 43,9 & 31,2 & $9,46(, 002)$ \\
\hline Sentir euforia & 47,7 & 38,3 & $\begin{array}{c}2,17 \\
(, 140)\end{array}$ & 47,8 & 32,2 & $13,96(, 000)$ \\
\hline $\begin{array}{l}\text { Facilidad para } \\
\text { hablar }\end{array}$ & 34,9 & 21,4 & $\begin{array}{l}5,86 \\
(, 015)\end{array}$ & 27,3 & 16,9 & $9,06(, 003)$ \\
\hline $\begin{array}{l}\text { Integrarme en } \\
\text { proyectos }\end{array}$ & 31,4 & 23,8 & $\begin{array}{c}1,82 \\
(, 177)\end{array}$ & 36,6 & 26,2 & $6,98(, 008)$ \\
\hline Sentir seguridad & 33,7 & 23,3 & $\begin{array}{c}3,39 \\
(, 065)\end{array}$ & 33,7 & 25,7 & $4,21(, 040)$ \\
\hline
\end{tabular}

Además, respecto a los incidentes experimentados a causa del alcohol, hay diferencias entre las personas en función de la edad de inicio, tanto en el caso de los hombres $\left(\mathrm{Chi}^{2}=20,09 ; p \leq, 001\right)$ como en el de las mujeres $\left(\mathrm{Chi}^{2}=20,09 ; \mathrm{p} \leq, 001\right)$, mostrando mayor prevalencia de consecuencias negativas aquellas personas que iniciaron antes, tal y como se observa en la figura 5 .

Figura 5. Porcentaje de hombres y mujeres que han sufrido consecuencias negativas a causa del consumo del alcohol, segun edad de inicio.

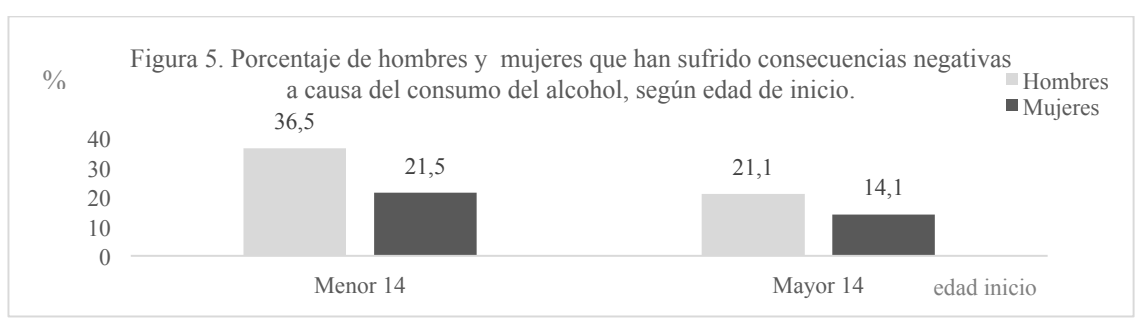

Sin embargo, en cuanto a la identificación del consumo de alcohol como un problema de salud, no existen diferencias estadísticamente significativas entre aquellas personas que iniciaron antes de los 14 años el consumo y las que lo hicieron después (ver tabla 6). La mayoría de ambos grupos, considera que no representa un programa importante y casi la mitad de los participantes, a excepción del grupo de hombres que iniciaron precozmente el consumo, reportan que no es un problema en absoluto. 
Tabla 6. Análisis diferenciales sobre la identificación del consumo de alcohol como un problema, en hombres y mujeres, según la edad de inicio de consumo

\begin{tabular}{ccccc}
\hline \multirow{2}{*}{ Frecuencia } & \multicolumn{3}{c}{ Hombre } & \multicolumn{2}{c}{ Mujer } \\
& Inicio antes 14 & Inicio tras 14 & Inicio antes 14 & Inicio tras 14 \\
\hline Nada & 37,6 & 49,5 & 50,2 & 57,3 \\
Poco & 47,1 & 38,2 & 37,9 & 34,8 \\
Bastante & 9,4 & 7,8 & 9,4 & 5,1 \\
Mucho & 5,9 & 4,4 & 2,5 & 2,8 \\
$\mathrm{Chi}^{2}(\mathrm{p})$ & \multicolumn{2}{c}{$3,42(, 331)$} & \multicolumn{2}{c}{$5,45(, 141)$} \\
\hline
\end{tabular}

\section{CONCLUSIONES}

En líneas generales, el estudio confirma la existencia de un patrón de consumo de alcohol de riesgo también entre la población joven universitaria, en línea con otros autores como Miquel et al. (2015). En este sentido, se observa en qué medida la mayoría de los participantes informan consumir alcohol y haber experimentado alguna borrachera en el último año, llegando a reconocer alrededor del $20 \%$ que ha sufrido consecuencias negativas debido al consumo. Además, en línea con Labhart et al. (2017), se cumplirían otros criterios de riesgo como el hecho de que las motivaciones del consumo se asocien a incrementar su integración social, mediar en el afrontamiento de sus problemas y aumentar su bienestar personal. Al mismo tiempo, algo menos de la mitad de los participantes reconoce que sea un problema, incrementando así las posibilidades de que el consumo se consolide (Espada, Pereira \& García-Fernández, 2008). Tal y como se observa en estudios previos (Carbia et al., 2016), los hombres parecen iniciar más tempranamente el consumo de alcohol y, en línea con Mota et al. (2010), también ellos mostrarían un mayor perfil de riesgo en cuanto a la frecuencia de consumo de las bebidas que resulta más elevada que entre las mujeres.

Por otro lado, tal y como señalan Carbia et al. (2016), existen diferencias notables en función de la edad en la que iniciaron el consumo. De hecho, aunque tanto los hombres como las mujeres que inician de manera más precoz el consumo, reconocen haber sufrido más consecuencias negativas derivadas de su uso, no existen diferencias notables en cuanto a la identificación del uso del alcohol como un problema, incrementando así la exposición al riesgo (Espada et al., 2008). De manera general, también se observa un mayor consumo de la mayoría de bebidas entre aquellas personas que iniciaron antes el hábito, siendo más frecuente también la experiencia de las borracheras, en la línea con lo que Mota et al. (2010) sostienen. En cuanto a las motivaciones, las diferencias también remarcarían un mayor perfil de riesgo entre las personas que iniciaron su consumo antes, pues resulta más frecuente que el uso de alcohol se asocie con motivos sociales o la mejora del bienestar personal, tanto en hombres como en mujeres (Labhart et al., 2017)

Cabría considerar algunas limitaciones en nuestro estudio. Por un parte y con el fin de poder generalizar los hallazgos, cabría ampliar la cantidad de participantes, así como incluir una mayor variabilidad entre los mismos, por ejemplo, en cuanto al nivel educativo. Por otro lado, sería importante tener en cuenta el carácter auto-informado del instrumento que podría facilitar la deseabilidad social y, en consecuencia, el sesgo de algunos resultados. Asimismo, en próximos estudios, sería interesante detallar algunos aspectos como el tipo de consecuencias negativas derivadas del uso del alcohol 0 graduar la identificación del consumo de alcohol como un problema. De esta forma, se podría desarrollar un análisis más pormenorizado de la percepción de riesgo asociado al alcohol.

En cualquier caso, por un lado, estos resultados ponen de manifiesto en qué medida el perfil de riesgo del consumo de alcohol está generalizado, a tenor de la prevalencia de su uso abusivo, el escaso reconocimiento del alcohol como un posible problema y la gran asimilación de aquellas 
motivaciones que, asociadas a la ingesta de alcohol, han sido relacionadas con una mayor exposición al riesgo. Por otro lado, se confirman las diferencias que podrían existir en el perfil de consumo de alcohol de la gente joven, en función de la edad en su inicio. En consecuencia, se visibiliza la importancia de profundizar los esfuerzos en la prevención primaria del consumo de alcohol tanto en hombres como en mujeres, con el fin de retrasar la edad de inicio, así como de prevenir el abuso.

\section{REFERENCIAS}

Abrams, K., Cieslowski, K., Johnson, S., Krimmel, S., Bierlein-De La Rosa, G., Barton, K., \& Silverman, P. (2018). The effect sof alcohol on heartbeat perception: Implications for anxiety. Addictive Behaviors, 79, 151-158.

Ballester, R. \& Gil, M.D (2009). ¿Por qué los jóvenes se dan atracones de alcohol los fines de semana? Estudio sobre creencias y actitudes relacionadas con ese patrón de consumo y diferencias de género. Revista de Psicopatología y Psicología Clínica, 14(1), 25-35.

Ballester, R., Gil, M.D. \& Guirado, M.C. (2000). Comportamientos y actitudes relacionados con el consumo de alcohol en adolescentes de 15 a 17 años. Análisis y Modificación de Conducta, 110, 855-898

Carbia, C., Corral, M., García, L.M., Cadaveira, F. \& Caamaño, F. (2016). Early alcohol use and psychopathological symptoms in university students. Psicothema, 28(3), 247-252.

Colder, C.R., Shyhalla, K. \& Frndak, S.E. (2018). Early alcohol use with parental permission: Psychosocial characteristics and drinking in late adolescence. Addictive Behaviors, 76, 82-87.

Dawson, D. A., Goldstein, R. B., Chou, S. P., Ruan, W. J., \& Grant, B. F. (2008). Age at first drink and the first incidence of adult-onset DSM-IV alcohol use disorders. Alcoholism, Clinical and Experimental Research, 32(12), 2149-2160.

Donoghue, K., Rose, H., Boniface, S., Deluca, P., Coulton, S., Alam, M. F., Gilvarry, E., Kaner, E., Lynch, E., Maconochie, I., McArdle, P., McGovern, R., Newbury-Birch, D., Patton, R., Phillips, C.J., Phillips, T., Russell, I., Strang, J. \& Drummond, C. (2017). Alcohol consumption, earlyonset drinking, and health-related consequences in adolescents presenting at emergency departments in England. Journal of Adolescent Health, 60(4), 438-446

Espada, J.P., Pereira, J.R., \& García-Fernández, J.M. (2008). Influencia de los modelos sociales en el consumo de alcohol de los adolescentes. Psicothema, 20(4), 531-537.

Gil, M.D. \& Ballester, R. (2002). Inicio temprano de consumo de alcohol entre niños de 9 a 14 años. Análisis y Modificación de Conducta, 118, 165-211.

Giménez-García, C. \& Ballester-Arnal, R. (2017). Cuestionario de Estilo de Vida y Salud para Población Joven. Manuscrito no publicado, Universitat Jaume I de Castellón, España.

Jacobus, J., \& Tapert, S. F. (2013). Neurotoxic effects of alcohol in adolescence. Annual Review of Clinical Psychology, 9, 703-721.

Labhart, F., Kuntsche, E., Wicki, M., \& Gmel, G. (2017). Reciprocal influences of drinking motives on alcohol use and related consequences: A full cross-lagged panel study among young adult men. Behavioral Medicine, 43(4), 277-284.

Lightowlers, C. (2017). Heterogeneity in drinking practices in England and Wales and its association with violent behavior: A latent class analysis. Substance Use \& Misuse, 52(13), 1721-1732.

Lin, P. (2016). Risky behaviors: Integrating adolescent egocentrism with the theory of planned behavior. Review of General Psychology, 20(4), 392-398.

Miquel, L., Rodamilans, M., Giménez, R., Cambras, T., Canudas, A.M. \& Gual, A. (2015). Evaluación del consumo de riesgo de alcohol en estudiantes universitarios de la Facultad de Farmacia. Adicciones, 27(3), 190-197. 


\section{ALCOHOL Y JUVENTUD ¿EXISTEN DIFERENCIAS EN CONSUMO DE HOMBRES Y MUJERES SEGÚN EDAD DE INICIO?}

Mota, N., Álvarez-Gil, R., Corral, M., Rodríguez, S., Parada, M., Crego, A., Caamaño-Isorna, F. \& Cadaveira, F. (2010). Risky alcohol use and heavy episodic drinking among Spanish University students: a two-year follow-up. Gaceta sanitaria, 24(5), 372-377.

Observatorio Español de Drogas y Adicciones (2017). Estadísticas 2017. Alcohol, tabaco y drogas ilegales en España. Recuperado de

http://www.pnsd.msssi.gob.es/profesionales/sistemasInformacion/informesEstadisticas/pdf/20170 EDA-INFORME.pdf

Plan Nacional Sobre Drogas (2018). Encuesta sobre uso de drogas en Enseñanzas Secundarias en España, ESTUDES. Recuperado de

http://www.pnsd.msssi.gob.es/profesionales/sistemas/nformacion/sistemalnformacion/encuestas_ESTUDES.htm

Secades-Villa, R., López-Núñez, C., Fernández-Artamendi, S., Weidberg, S. \& Fernández-Hermida, J.R. (2013). Gender differences in the prevalence of DSM-IV alcohol use disorders in adolescents. Adicciones, 25(3), 260-268.

West, R. K., Maynard, M. E., \& Leasure, J. L. (2018). Binge ethanol effects on prefrontal cortex neurons, spatial working memory and task-induced neuronal activation in male and female rats. Physiology \& Behavior, 188, 79-85.

White, A., \& Hingson, R. (2014). The burden of alcohol use: Excessive alcohol consumption and related consequences among college students. Alcohol Research: Current Reviews, 35(2), 201218. 